PROCEEDINGS OF THE

AMERICAN MATHEMATICAL SOCIETY

Volume 134, Number 10, October 2006, Pages 2881-2884

S 0002-9939(06)08046-4

Article electronically published on May 9, 2006

\title{
A THEOREM ON REFLEXIVE LARGE RANK OPERATOR SPACES
}

\author{
LIFENG DING
}

(Communicated by David R. Larson)

\begin{abstract}
If every nonzero operator in an $n$-dimensional operator space $\mathbb{S}$ has rank $\geqslant 2 n$, then $\mathbb{S}$ is reflexive.
\end{abstract}

Let $\mathbb{V}$ be a vector space over a field $\mathbb{F}$, and let $\mathcal{L}(\mathbb{V})$ be the space of all linear operators on $\mathbb{V}$. Suppose $\mathbb{S}$ is a finite-dimensional subspace of $\mathcal{L}(\mathbb{V})$. The reflexive closure of $\mathbb{S}$ is given by

$$
\text { ref } \mathbb{S}=\{t \in \mathcal{L}(\mathbb{V}): t x \in \mathbb{S} x \text { for all } x \in \mathbb{V}\},
$$

where $\mathbb{S} x=\{s x: s \in \mathbb{S}\}$, which is called the cyclic subspace. We note that every element $t$ of ref $\mathbb{S}$ interpolates $\mathbb{S}$, or $t$ is locally in $\mathbb{S}$. It is obvious that $\mathbb{S} \subseteq$ ref $\mathbb{S}$. If $\mathbb{S}=\operatorname{ref} \mathbb{S}$, then $\mathbb{S}$ is said to be reflexive. The reflexivity problem was originally introduced as a topological notion by P. Halmos to describe those operator algebras that are determined by their closed invariant subspace lattices of a Hilbert space. Loginov and Sulman extended reflexivity to include operator subspaces which are not necessarily algebras. The purely algebraic version of reflexivity and a unified approach was introduced by Hadwin [4, 5]. When the operator subspace is finite dimensional the topological version and the algebraic version of reflexivity coincide. The reflexivity problem is far from being complete. A historical account can be found in [1, 2, 4, 5, 6, 8].

A well-known result of Larson [6] asserts that for the finite-dimensional $\mathbb{S}$, ref $\mathbb{S}=\mathbb{S}+\operatorname{ref} \mathbb{S}_{F}$, where $\mathbb{S}_{F}$ is the collection of all finite rank operators in $\mathbb{S}$. Hence if $\mathbb{S}_{F}=\{0\}$, then $\mathbb{S}$ is reflexive. In 2, 3] we further find that if the rank of each nonzero operator in $\mathbb{S}$ is large enough, then $\mathbb{S}$ is reflexive. For example, in 2 we proved that if the dimension of $\mathbb{S}$ is $n$, and every nonzero operator of $\mathbb{S}$ has rank $>2 n^{2}-n$, then $\mathbb{S}$ is reflexive. This result was improved to that if every nonzero operator of $\mathbb{S}$ has rank $>n^{2}$, then $\mathbb{S}$ is reflexive 3 . In this paper we lower the rank to $2 n$. This is achieved by making full use of Proposition 1.1 in [3] to prove a generalized version of Theorem 2.1 in [3] and using Theorem 1.2 in [3].

A vector $x$ is a separating vector for $\mathbb{S}$ if the map $\mathbb{S} \rightarrow \mathbb{S} x$ by sending $s$ to $s x$ is injective. A linear subspace $\mathbb{M}$ of $\mathbb{V}$ is said to be a separating subspace for $\mathbb{S}$ if the only member $s$ of $\mathbb{S}$ satisfying $s(\mathbb{M})=\{0\}$ is $s=0$. It is easy to see that if $\mathbb{S}$ has a separating vector $x$, then any subspace containing $x$ is a separating subspace for $\mathbb{S}$. However, if the dimension of a separating subspace is greater than 1 , then $\mathbb{S}$ may

Received by the editors May 2, 2001 and, in revised form, November 8, 2004.

2000 Mathematics Subject Classification. Primary 47L05; Secondary 15A04.

Key words and phrases. Reflexive operator space, separating vector.

(C)2006 American Mathematical Society

Reverts to public domain 28 years from publication 
not have a separating vector. Trivially, $\mathbb{V}$ is a separating subspace for $\mathbb{S}$. Of course, if $\mathbb{M}$ is a separating subspace for $\mathbb{S}$, then any subspace containing $\mathbb{M}$ is a separating subspace for $\mathbb{S}$. In [3] we proved the following theorem:

Theorem 1 (Theorem 1.2, [3]). Suppose the linear subspace $\mathbb{S} \subseteq \mathcal{L}(\mathbb{V})$ has a separating vector $x$ and a separating subspace $\mathbb{M}$ satisfying

(1) $\operatorname{dim} \mathbb{S}<$ cardinality of the field $\mathbb{F}$,

(2) $\mathbb{S} x \cap \mathbb{S}(\mathbb{M})=\{0\}$.

Then $\mathbb{S}$ is reflexive.

Proposition 2 (Proposition 1.1, 3]). Suppose $\mathbb{S}$ is a linear subspace of $\mathcal{L}(\mathbb{V})$, and the dimension of $\mathbb{S}$ is less than the cardinality of $\mathbb{F}$. Let $x$ be a separating vector for $\mathbb{S}$, and let $\mathbb{W}$ be a linear subspace of $\mathbb{V}$ satisfying $\mathbb{S} x \cap \mathbb{W}=\{0\}$. Then for each vector $y \in \mathbb{V}$ there is a scalar $\lambda \in \mathbb{F}$ such that $y+\lambda x$ is a separating vector for $\mathbb{S}$ and $\mathbb{S}(y+\lambda x) \cap \mathbb{W}=\{0\}$.

The proof of the proposition is obtained by using the mapping $\phi=E_{x}^{-1} \cdot P \cdot E_{y}$ on $\mathbb{S}$ where $E_{y}$ is an evaluation map, and $P$ is a projection with range $\mathbb{S} x$, and the fact that the number of different eigenvalues of $\phi$ is less than the cardinality of the field $\mathbb{F}$. The next Theorem 3 is a generalized version of Theorem 2.1 in 3 .

Theorem 3. Suppose $\mathbb{V}$ is a vector space over a field $\mathbb{F}$. Let $\mathbb{W}$ be a finitedimensional linear subspace of $\mathbb{V}$, and let $\mathbb{S}$ be an $n$-dimensional linear subspace of $\mathcal{L}(\mathbb{V})$, where $n$ is less than the cardinality of the field $\mathbb{F}$. If every nonzero operator in $\mathbb{S}$ has rank greater than or equal to $n+\operatorname{dim} \mathbb{W}$, then $\mathbb{S}$ has a separating vector $x$ such that $\mathbb{S} x \cap \mathbb{W}=\{0\}$.

Proof. We proceed by induction on the dimension $n$. For $n=1, \mathbb{S}=\operatorname{span}\{s\}$, and rank of $s \geqslant 1+\operatorname{dim} \mathbb{W}$. Choose a vector $y \in \operatorname{range}$ of $s$ and $y \notin \mathbb{W}$. Let $s x=y$. Then $x$ separates $\mathbb{S}$ and $\mathbb{S} x \cap \mathbb{W}=\{0\}$.

Assume the statement is true for each $(n-1)$-dimensional subspace of $\mathcal{L}(\mathbb{V})$. We consider $n$-dimensional $\mathbb{S}$. Let $s_{1}, \cdots, s_{n}$ be a basis of $\mathbb{S}$. Denote by $\mathbb{S}^{\prime}$ the linear span of $\left\{s_{1}, \cdots, s_{n-1}\right\}$. By the induction assumption we get a separating vector $x$ for $\mathbb{S}^{\prime}$ and $\mathbb{S}^{\prime} x \cap \mathbb{W}=\{0\}$.

If $s_{n} x \notin$ linear span $\left\{\mathbb{S}^{\prime} x, \mathbb{W}\right\}$, then for any scalars $\alpha_{1}, \cdots, \alpha_{n-1}, \alpha_{n} \in \mathbb{F}$, with $\alpha_{1} s_{1} x+\cdots+\alpha_{n-1} s_{n-1} x+\alpha_{n} s_{n} x \in \mathbb{W}$, we have $\alpha_{n} s_{n} x=z-\left(\alpha_{1} s_{1} x+\cdots+\right.$ $\left.\alpha_{n-1} s_{n-1} x\right)$ for some $z \in \mathbb{W}$. Since $s_{n} x \notin$ linear span $\left\{\mathbb{S}^{\prime} x, \mathbb{W}\right\}, \alpha_{n} s_{n} x=0$, and $z-\left(\alpha_{1} s_{1} x+\cdots+\alpha_{n-1} s_{n-1} x\right)=0$. It follows that $\alpha_{n}=0$, and $z=0$ and $\alpha_{1} s_{1} x+\cdots+\alpha_{n-1} s_{n-1} x=0$, since $\mathbb{S}^{\prime} x \cap \mathbb{W}=\{0\}$. Then $\alpha_{1}=\cdots=\alpha_{n-1}=0$. This proves that $\mathbb{S} x \cap \mathbb{W}=\{0\}$, and $s_{1} x, \cdots, s_{n-1} x, s_{n} x$ are linearly independent, and hence $x$ is a separating vector for $\mathbb{S}$. The proof of the theorem is done in this case. So we need only consider the case that $s_{n} x \in \operatorname{linear} \operatorname{span}\left\{\mathbb{S}^{\prime} x, \mathbb{W}\right\}$.

Let $s_{n} x=\left(\alpha_{1} s_{1} x+\cdots+\alpha_{n-1} s_{n-1} x\right)+w$, for some scalars $\alpha_{1}, \cdots, \alpha_{n-1} \in \mathbb{F}$, and $w \in \mathbb{W}$. Replacing $s_{n}$ by $s_{n}^{\prime}=s_{n}-\alpha_{1} s_{1}-\cdots-\alpha_{n-1} s_{n-1}$, we may assume $s_{n} x \in \mathbb{W}$.

Since rank of $s_{n} \geqslant n+\operatorname{dim} \mathbb{W}$, there is $y \in \mathbb{V}$ such that $s_{n} y \notin$ linear span of $\left\{\mathbb{S}^{\prime} x, \mathbb{W}\right\}$. Note that $\mathbb{S}^{\prime} x \cap \mathbb{W}=\{0\}$, so this also implies that $\mathbb{S}^{\prime} x \cap$ linear span $\left\{s_{n} y, \mathbb{W}\right\}=\{0\}$. By Proposition 2, there is scalar $\lambda$ so that $y+\lambda x$ separates $\mathbb{S}^{\prime}$, and $\mathbb{S}^{\prime}(y+\lambda x) \cap$ linear span $\left\{s_{n} y, \mathbb{W}\right\}=\{0\}$. We next claim $y+\lambda x$ is a separating vector for $\mathbb{S}$. 
Let $t \in \mathbb{S}^{\prime}$ and $\alpha \in \mathbb{F}$ with $\left(t+\alpha s_{n}\right)(y+\lambda x)=0$. Then

$$
t(y+\lambda x)=-\alpha s_{n}(y+\lambda x)=-\alpha s_{n} y-\alpha \lambda s_{n} x,
$$

where $\alpha \lambda s_{n} x \in \mathbb{W}$. By the disjointness of $\mathbb{S}^{\prime}(y+\lambda x)$ and linear span $\left\{s_{n} y, \mathbb{W}\right\}$, we have $t(y+\lambda x)=0$, and $\alpha s_{n} y=-\alpha \lambda s_{n} x$. Hence $t=0$, and $\alpha=0$, since $s_{n} y \notin \mathbb{W}$. This completes the proof of the claim.

Finally, we show that $\mathbb{S}(y+\lambda x) \cap \mathbb{W}=\{0\}$. Indeed, suppose there are scalars $\alpha_{1}, \cdots, \alpha_{n}$ satisfying

$$
\alpha_{1} s_{1}(y+\lambda x)+\cdots+\alpha_{n-1} s_{n-1}(y+\lambda x)+\alpha_{n} s_{n}(y+\lambda x) \in \mathbb{W}
$$

Then $\alpha_{1} s_{1}(y+\lambda x)+\cdots+\alpha_{n-1} s_{n-1}(y+\lambda x)+\alpha_{n} s_{n}(y+\lambda x)=z$ for some $z \in \mathbb{W}$. It follows that

$$
-\alpha_{n} s_{n} y=\left(\alpha_{1} s_{1}(y+\lambda x)+\cdots+\alpha_{n-1} s_{n-1}(y+\lambda x)\right)+\left(\alpha_{n} \lambda s_{n} x-z\right) .
$$

Since $\alpha_{n} \lambda s_{n} x-z \in \mathbb{W}$ and $s_{n} y \notin$ linear span of $\left\{\mathbb{S}^{\prime} x, \mathbb{W}\right\}$, we conclude that $\alpha_{n} s_{n} y=0$. It follows that $\alpha_{n}=0$. Again, the disjointness of $\mathbb{S}^{\prime}(y+\lambda x) \cap \mathbb{W}=\{0\}$ implies that $z=0$, and $\alpha_{1} s_{1}(y+\lambda x)+\cdots+\alpha_{n-1} s_{n-1}(y+\lambda x)=0$. Therefore,

$$
\alpha_{1}=\cdots=\alpha_{n-1}=0 \text {. }
$$

Before stating our next result we set the notation below. If $\mathbb{M}$ is a subspace of $\mathbb{V}$, we write $\mathbb{S}(\mathbb{M})$ for the linear span of $\{s(\mathbb{M}): s \in \mathbb{S}\}$. We also assume that the cardinality of the field $\mathbb{F}$ is greater than the dimension of $\mathbb{S}$. Combining Theorem 3 and Theorem 1, we immediately have the following conclusion.

Theorem 4. Let $\mathbb{V}$ be a vector space over a field $\mathbb{F}$ and $\mathbb{S}$ an $n$-dimensional subspace of $\mathcal{L}(\mathbb{V})$. Suppose $\mathbb{M}$ is a separating subspace for $\mathbb{S}$ and $\mathbb{S}(\mathbb{M})$ is finite dimensional. If every nonzero operator in $\mathbb{S}$ has rank greater than or equal to $n+\operatorname{dim} \mathbb{S}(\mathbb{M})$, then $\mathbb{S}$ is reflexive.

Proof. By Theorem 3 there is a separating vector $x$ for $\mathbb{S}$ such that $\mathbb{S} x \cap \mathbb{S}(\mathbb{M})=\{0\}$. The conclusion follows immediately from Theorem 1.

In [2, 3] we actually obtained several separating subspaces. For example, in Theorem 2.3 3 we assume every nonzero operator in $\mathbb{S}$ has rank greater than $n^{2}$. Then $\mathbb{S}$ has a separating vector $x$. Let $\mathbb{W}$ be a vector space complement of $\mathbb{S} x$ in $\mathbb{V}$. Then $\mathbb{U}=\{u \in \mathbb{V}: \mathbb{S} u \subseteq \mathbb{W}\}$ is a separating subspace for $\mathbb{S}$. Theorem 4 opens a door to lower the rank requirement. A much better improvement of lowering the rank is ready now. Before we state the improved result we give a corollary of Theorem 3 .

Corollary 5. Let $\mathbb{V}$ be a vector space over a field $\mathbb{F}$ and suppose $\mathbb{S}$ is an $n$ dimensional linear subspace of $\mathcal{L}(\mathbb{V})$, where $n$ is less than the cardinality of the field $\mathbb{F}$. Then if every nonzero operator in $\mathbb{S}$ has rank $\geqslant n$, then $\mathbb{S}$ has a separating vector.

Proof. In Theorem 3 we choose $\mathbb{W}=\{0\}$. The proof follows.

Remark 6. This corollary recovers Theorem 2.1 of [3].

Theorem 7. Let $\mathbb{V}$ be a vector space over a field $\mathbb{F}$. Suppose $\mathbb{S}$ is an $n$-dimensional linear subspace of $\mathcal{L}(\mathbb{V})$, where $n$ is less than the cardinality of the field $\mathbb{F}$. Then, if every nonzero operator in $\mathbb{S}$ has rank $\geqslant 2 n$, then $\mathbb{S}$ is reflexive. 
Proof. By Corollary 5 there is a separating vector $x$ for $\mathbb{S}$. Then the cyclic subspace $\mathbb{S} x$ has dimension $n$. Note that the separating vector $x$ generates a one-dimensional separating subspace for $\mathbb{S}$, and this matches the assumption of $\mathbb{S}(\mathbb{M})$ of Theorem 4 . Hence the proof is completed.

Remark 8 . In the proof we see that if the rank $\geqslant 2 n$, then we obtain a disjoint pair of separating vectors which provides an example for the main result Theorem 2.1 in 2 .

Remark 9. The contrapositive of Corollary 5 states that for $n$ linear operators $s_{1}, \cdots, s_{n}$ on $\mathbb{V}$, if $s_{1} u, \cdots, s_{n} u$ are linearly dependent for every vector $u \in \mathbb{V}$, then there are scalars $\alpha_{1}, \cdots, \alpha_{n}$, not all zero, such that $s=\alpha_{1} s_{1}+\cdots+\alpha_{n} s_{n}$ has rank $\leqslant n-1$. As pointed out in Remark 2.2 of 3 this is Aupetit's well-known improvement of Kaplansky's lemma which states that if a linear operator on a linear space is locally algebraic, and the degrees of the related polynomials form a bounded set, then the operator is algebraic.

Remark 10. After this paper was accepted, J. Li and Z. Pan [7] showed that $2 n$ can be reduced to $2 n-1$ in Theorem 6 when the scalar field is $\mathbb{C}$.

\section{REFERENCES}

[1] E. A. Azoff, On finite rank operators and preannihilators, Memoirs Amer. Math. Soc. 357 (1986). MR0858467 (88a:47041)

[2] L. Ding, Separating vectors and reflexivity, Lin. Alg. Appl. 174 (1992), 37-52. MR.1176449 (94a:47075)

[3] L. Ding, On a pattern of reflexive operator spaces, Proc. Amer. Math. Soc. 124 (1996), 31013108. MR 1343689 (97h:47039)

[4] D. Hadwin, Algebraically reflexive linear transformations, Lin. Multilin. Alg. 14 (1983), 225233. MR 0718951 (85e:47003)

[5] D. Hadwin, A general view of reflexivity, Trans. Amer. Math. Soc. 344 (1994), 325-360. MR.1239639 (95f:47071)

[6] D. R. Larson, Reflexivity, algebraic reflexivity, and linear interpolation, Amer. J. Math. 110 (1988), 283-299. MR0935008 (89d:47096)

[7] J. Li and Z. Pan, Reflexivity and hyperreflexivity of operator spaces, Math. Anal. Appl. 279 (2003), 210-215. MR1970501 (2004a:47001)

[8] H. Radjavi and P. Rosenthal, Invariant Subspaces, Springer-Verlag, 1973. MR0367682 $(51: 3924)$

Department of Mathematics and Statistics, Georgia State University, Atlanta, GEORGIA 30303-3083

E-mail address: matlfd@panther.gsu.edu 